Für die Bibliothek ist als Geschenk eingegangen:

773. Sammlung chemischer und ehemisch-technischer Vorträge, herausgegeben von F. B. Ahrens. XI. Band, Heft 8/9: Fr. N. Schulz, Allgemeine Chemie der Eiweißstoffe. Stuttgart 1907.
Der Vorsitzende:
Der Schriftführer:
S. Gabriel.
C. Schotten.

\title{
Mitteilungen.
}

\section{W. Tschelinzeff: Die Umwandlung Individueller magnesiumorganischer Verbindungen in Aminkomplexe und ihre thermochemische Untersuchung".}

(Eingegangen am 11. März 1907.)

Bei der Einwirkung von gewöhnlichem Äther auf individuelle magnesiumorganische Verbindungen vom Typus R.MgJ vollzieht sich, wie ich zu berichten Gelegenheit hatte '), eine stürmische Reaktion, durch welche sich die bekannten Grignardschen Komplexe bilden; ihre Zısammensetzung wird nach meinen Untersuchungen ${ }^{2}$ ) durch die Formel RMgJ .2 $\left(\mathrm{C}_{2} \mathrm{H}_{3}\right)_{3} \mathrm{O}$ ausgedrückt. Einer ganzen Reibe von Erwägungen zufolge muß diesen Verbindungen, nach meiner Meinung, eine Struktur nach dem Oxoniumtypus zugeschrieben werden, was ich in allgemeiner Form in meinen letzten Mitteilungen entwickelt habe ${ }^{3}$ ); betreffs näherer Einzelheiten in der Struktur jedoch verlangt diese Frage eine eingehendere experimentelle Beleuchtung. In weiteren Berichten werde ich noch hierauf zurückkommen; ich will jetzt nur bemerken, daß zu Gunsten der Oxoniumtheorie auch die Analogie der nämlicheu Verbindungen mit den eben von mir erhaltenen neuen Verbindungen rom Ammoniumtypus spricht; einer Beschreibung der letzteren ist diese Abhandlung gewidmet.

Schon in meinem ersten Berichte ${ }^{t}$ ) habe ich auf die Neigung der iudividuellen magnesiumorganischen Verbindungen zur Bildung von

1) Diese Berichte 38, 366t [1905]; Journ. d. Russ. Phys.-chem. Ges. 37, 1100 .

2) Diese Berichte 39, 773 [1906]: Journ. d. Russ. Phys.-chem. Ges. 38, 579.

3) Compt. rend. 143, 1237 [1906]; 144, 88 [1907].

4) Diese Berichte 87, 2081, 4534 [1904]: Journ. d. Kuss. Phys.-chem. Ges. 36, 1268: 37, 367. 
Ammoniumverbivdungen, die den Äther-Oxoniumverbindungen ähnlich sind, bingewiesen. Seitdem ist die Literatur über diese Frage durch einige Daten bereichert worden: so haben F. Sachs und L. Sachs') nachgewiesen, daß magnesiumorganische Verbindungen Komplexe mit Pyridin und ('hinolin geben; gleichzeitig hat B. Oddo ${ }^{2}$ ) über die Bildung von den letzteren nahestehenden Komplexen berichtet, seine Polemik mit L. und F. Sachs über die nähere Zusammeusetzung dieser Komplexe ist jedoch noch nicht entschieden; weiter hat J. Houben ${ }^{3}$ ) noch kompliziertere Komplexe magnesiumorganischer Verbindungen mit den Meunierschen Verbindungen beschrieben; in letzter Zeit berichtete M. Popoff') über Komplexe der nämlichen Meunierschen magnesiumorganischen Verbindungen mit primären Aminen, und endlich wurden von Senier, Austin und Clarke ${ }^{3}$ ) noch kompliziertere Verbindungen, nämlich mit Acridin, die möglicherweise von derselben Art sind, beschrieben. Alle genannten Autoren erhielten jedoch ihre Magnesium-Ammoniumverbindungen aus magnesiumorganischen Ätherkomplexen, d. h. durch Substitution der Äther mittels organischer Stickstoffverbindungen; in meinen Versuchen dagegen erhielt ich die Verbindungen vom Ammoniumtypus direkt aus den individuellen magnesiumorganischen Verbindungen, also durch die Reaktion der direkten Anlagerung stickstoffhaltiger Körper an letztere. Diese Methode hat zwei Vorzüge: erstens erlaubt sie nicht nur, Komplexe zu erhalten, deren Bildungswärme aus den nächsten Komponenten eine größere ist, als die der Ätherkomplexe, sondern auch solche, bei denen sie eine kleinere ist; zweitens haben wir bei der Anwendung dieser Methode mehr Aussicht auf ein reines Produkt, denn bei gleichzeitiger Gegenwart von Äther und Amin könnte man, wenigstens in manchen Fällen, eine Verteilung der individuellen magnesiumorganischen Verbindungen zwischen Äther und Amin erwarten, wodurch eine Mischung von Oxoniun- und Ammoniumverbindungen erhalten werden würde.

Zur Darstellung der Magnesium-Ammoniumverbindungen nahm ich anfangs die einfachsten Stickstoffverbindungen vom Typus $\mathrm{NR}_{\text {; }}$, wo $\mathbf{R}$ entweder fette oder aromatische Radikale waren, oder auch kompliziertere Stickstoffverbindungen, doch ebenfalls tertiärer Natur, wie z. B. Pyridin und Chinolin; da jedoch die Verhältnisse der letzteren zu den individuellen magnesiumorganischen Verbindungen, besonders.

\footnotetext{
3) Diese Berichte 37, 3088 [1904].

2) Atti R. Accad. dei Lincei Roma [5] 13, 1I, 100: Zentralbl. 1904, II, 836 .

3) Diese Berichte 88, 3017 [1905].

4) Journ. d. Russ. Phys.-chem. Ges. 38, 681.

5) Proc. Chem. Soc. 21, 227; Journ. Chem. Soc. 87, 1469.
} 
bei thermochemischen Lintersuchungen, ganz verschiedene sind von denjenigen der einfachsten tertiären Amine, so sah ich mich genötigt, sie in eine besondere Gruppe einzuordnen; infolgedessen beschältige ich mich in meinen Berichten ausschließlich mit Komplexen mit einfachen tertiären Aminen von Typus $\mathrm{NR}_{3}$, deren Stickstoffaton bestimmtere Funktionen aufweist.

Aminkomplexe dieser Art werden älnlich den Ätherkomplexen durch einfache Einwirkung der tertiären Amine auf individuelle magnesiumorganische Verbindungen unter gewöhnlichen Bedingungen erhalten. Sie bilden ihrem Äußeren nach feste, lockere, weiße Körper, die bei niederen fetten Aminen in Benzol leicht löslich, in Benzin dagegen unlöslich sind, bei höheren fetten oder aromatischen Aminen aber entweder schwer oder sogar ganz unlöslich sind, sowohl in Benzin, als auch in Benzol. Ihre chemischen Eigenschaften im Sinne ihrer Einwirkung auf carbonylorganische Verbindungen, Wasser usw., scheinen ïberhaupt keinen Unterschied von einer ähnlichen Einwirkung der Ätherkomplexe aufzuweisen, wodurch ihre priuzipielle Analogie mit den letzteren nachgewiesen wird. Diese Ähnlichkeit ließe sich auch in der Nomenklatur zum Ausdruck bringen, indem man den Aminand den Ätherkomplexen analoge Benennungen gibt; behält man für letztere die Benennung »Ätherate《 bei, so müßte man erstere »Aminat e« nennen.

Beide Reihen von Komplexen weisen jedoch auch einige Unterschiede auf, an erster Stelle in ihrer Zusammensetzung. Können bei gewöhnlichen Bedingungen zu den individuellen magnesiumorganischen Verbindungen ein oder zwei Moleküle der Äther addiert werden, so treten die tertiären Amine nur in der Zahl von einem Molekül mit ihnen in Verbindung, so daß die Form der hieraus entstehenden Aminate durch

$$
\mathrm{RMgJ} \cdot \mathrm{NR}_{3}
$$

zum Ausdruck gebracht wird.

Bis jetzt ist es schwer zu sagen, weshalb weniger Anin als Äther in Verbindung tritt; man kann jedoch die Bemerkung nicht unterlassen, daß bei der Bildung ron Komplexen mit Stickstoffiverbindungen überhaupt sehr oft weniger Stickstoffkörper in Verbiudung tritt, als dies bei Sauerstoffverbindungen der Fall wäre. Hierauf begründet sich auch der Satz Werners') über die Bestimmung seiner "Koordinationszahl" aus der Zusammensetzung der Ammoniak- und Aminkomplexe; diese Zahl kann jedoch nicht aus der Formel von, beispiels-

1) A. Werner, "Neuere Anschauungen auf dem Gebiete der anorgan. Chemier, S. 110 [1905]. 
weise, Hydraten begründet werden, wo die Anzahl der Wassermoleküle die »Koordinationszahl《 oft übertrifft.

Betreffs der Konstitution dieser "Aminate" stellen sich uns zwei Möglichkeiten entgegen, die durch folgende Schemata zum Ausdruck gebracht werden:

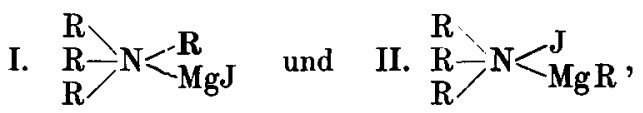

denjenigen vollständig analog, die wir bei den Monoätheraten hatten. Natürlich sind das, wie auch bei den Ätheraten, nicht die einzigen theoretisch möglichen Anlagerungsformen von RMgJ an tertiäre Amine, jedoch meiner Meinung nach die wahrscheinlichsten. In meinem ersten Artikel über diesen Gegenstand ${ }^{1}$ ) hatte ich mich enthalten, diese Verbindungen durch eine Konstitutionsformel auszudrücken, denn der Ausdruck, den ich anfangs im Sinne der ersten Formel in den Protokollen der Russ. Phys.-chem. Ges. zur vorläufigen Orientierung gegeben hatte, schien mir damals wenig zufriedenstellend. Später jedoch, als mir nach meinen weiteren Untersuchungen die Oxoniumformel von Baeyer und Villiger für Ätherkomplexe wahrscheinlicher geworden war, schien mir auch für die Aminkomplexe die Formel vom Typus II entsprechender ${ }^{2}$ ).

1) Diese Berichte 37, 4534 [1904].; Journ. d. Russ. Phys.-chem. Ges. 37, 367.

2) In diesem Sinne wurde es auch z. B. von Hrn. J. B. Tingle aufgefabt, wie aus seiner Übersicht meiner Arbeiten im "Amer. Chem. Journ." (Bd. 35, S. 94) zu ersehen ist; trotzdem hatte ich bisher bei meinen Untersuchungen der Ätherkomplexe noch nicht Gelegenheit gehabt, darauf hinzuweisen, was auch gerechterweise die Anfechtungen von Seiten von Blaise nach sich zog. (Bull. Soc. Chim. [3] 35, 90.) Doch bin ich mit der Meinung von B la is e nicht einverstanden, daß die Wahl zwischen diesen Formeln auf Grund der Zersetzung der Aminkomplexe durch Wasser zu treffen sei, denn die Zersetzung durch Wasser muß nach beiden Formeln zu gleichen Produkten führen:

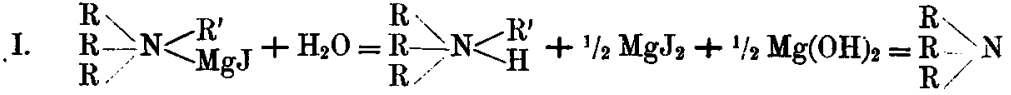
$+\mathrm{R}^{\prime} \mathrm{H}+1 / 2 \mathrm{MgJ}_{2}+1 / 2 \mathrm{Mg}(\mathrm{OH})_{2}$

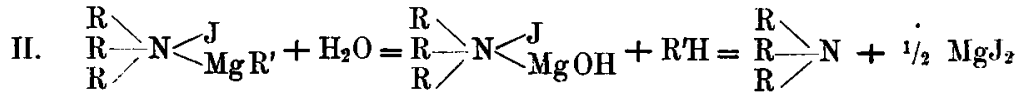
$+1 / 2 \mathrm{Mg}(\mathrm{OH})_{2}+\mathrm{RH}_{2}$

nnd auf keine Weise kann man, meiner Meinung nach, $R_{4} N . O H$ erhalten, wie Blaise vermutet. Dessen ungeachtet hat Blaise rollkommen Recht, daß man den Magnesium-Ammoniumverbindungen, den Oxoniumverbindungen analog, die Formel vom Typus II zuschreiben müsse; diese meine schon. längst gefaßte Meinung kann ich jetzt durch Experimente bekräftigen, die ich in meinen năchsten Artikeln beschreiben werde. 
Geht man von dieser Formel aus, so wird der ProzeB der Anlagerung individueller magnesiumorganischer Verbingungen an tertiäre Amine erstens in der Zerspaltung der magnesiumorganischen Verbindungen in zwei Gruppen - J und $\mathrm{MgR}$ - und zweitens in der Anlagerung dieser beiden Gruppen an das Stickstoffatom des Amins bestehen. Die bei dieser Operation entstehende Wärme wird die s'Bildungswärme der Magnesium-Ammoniumverbindungen aus ihren nächsten Komponenten " ausdrücken. Bezeichnet man diese Wärme durch $Q$, so wird der ganze Prozeß durch folgende thermische Gleichung ausgedrückt:

$$
[\mathrm{RMgJ}]+\mathrm{NR}_{3}=\mathrm{RMgJ} \cdot \mathrm{NR}_{3}+\mathrm{Q},
$$

wo das $\mathbf{R}$ der magnesiumorganischen Verbindung in allen folgenden Versuchen ein und dasselbe Radikal bezeichnet, $\mathbf{N R}_{3}$ jedoch in verschiedenen Versuchen auch verschiedene Amine, sowohl mit fetten, als auch mit aromatischen Radikalen.

Diese Untersuchung soll also den Einfluß der Radikale auf die Natur des Stickstoffatoms der Amine zeigen, und die Vergleichung ihrer Resultate mit denjenigen meiner früheren ähnlichen Untersuchung in der Reihe der Oxoniumverbindungen soll klarstellen, wie weit eine Analogie zwischen dem Sauerstoff- und den Stickstoffverbindungen im Sinne des Einflusses der verschiedenen organischen Radikale auf ihre Zentralatome - Stickstoff und Saverstoff -, wenn ihre überschüssigen Valenzen zum Vorschein kommen, zu ziehen ist; sie soll auch die relativen Energiemengen zeigen, die in Gestalt von Wärme bei der Bildurg analoger Oxonium- und Ammoniumverbindungen abgegeben werden.

\section{Experimenteller Teil.}

Der Prozeb der Umwandlung individueller magnesiumorganischer Verbindungen in Verbindungen vom Ammoniumtypus gebt bei Anwesenheit folgender Körper vor sich: 1) individ. magnesiumorganische Verbindungen,'2) tertiäres Amin, 3) Benzol. Zur Charakteristik der Versuche ist es unerläßlich, über jeden dieser Stoffe einige Bemerkungen zu machen.

Als individuelle magnesiumorganische Verbindung diente, wie schon oben bemerkt, ein und derselbe Körper, nämlich das Propylmagnesiumjodid die Wahl dieses Körpers erklärt sich dadurch, daß er unter allen einfacheren magnesiumorganischen Verbindungen am leichtesten und am reinsten zu erhalten ist; die quantitative Seite der Darstellung von $\mathrm{C}_{3} \mathrm{H}_{7} \mathrm{MgJ}$ läßt sich nach seiner Zersetzung durch Wasser beurteilen. Folgende zwei Analysen können eine Vorstellung von dieser Seite der Untersuchung geben. 
I. Zur Darstellung von $\mathrm{C}_{3} \mathrm{H}_{7} \mathrm{Mg} \mathrm{J}$ warde genommen: $0.6132 \mathrm{~g} \mathrm{Mg}$ (mit Berücksichtigung auf Beimischungen in der Menge ron $0.7 \%$ ).

Nach der Zersetzung der magnesiumorganischen Verbindung durch $W_{\text {asser }}$ wurden $606 \mathrm{ccm}$ trocknen Gases bei $19^{\circ}$ und $750 \mathrm{~mm}$ Drnck erhalten.

Das Gas auf normale Bedingungen reduziert ergibt $.559 .0 \mathrm{ccm}$ :

Die Theorie erfordert aber für die oben genannte Quantität $\mathrm{C}_{3} \mathrm{H}_{7} \mathrm{Mg} . \mathrm{J}$ $572.8 \mathrm{ccm}$ Gas. Unterschied $2.4 \%$.

II. $\mathrm{Zur}$ Darstellung von $\mathrm{C}_{3} \mathrm{H}_{7} \mathrm{MgJ}$ wurde genommen: $0.6028 \mathrm{~g} \mathrm{Mg}$ (Beimischungen: $0.7 \%$ ).

Nach Zersetzung der magnesiumorganischen Verbindung durch Wasiner erhielt man $604 \mathrm{ccm}$ trocknes Gas bei $20^{\circ}$ und $746 \mathrm{~mm}$ Druck.

Das Gas auf normale Bedingungen reduziert ergibt $552.3 \mathrm{ccm}$.

Die Theorie erfordert aber für die oben genannte Quantităt $\mathrm{C}_{3} \mathrm{H}_{7} \mathrm{Mg}$.J $563.1 \mathrm{ccm}$ Gas. Unterschied $2.0 \%$.

Die Einzelheiten der Darstellung magnesiumorganischer Verbindungen habe ich schon früher beschrieben I); es ist nur zu bemerken, daß hierbei ein Überschuß von Jodid genommen wurde, was insofern wichtig ist, da bei diesen Versuchen in die calorimetrische Kammer tertiäres Amin bineingebracht wurde; es entsteht also die Frage, (1) das bei der Zubereitung der magnesiumorganischen Verbindung übrig bleibende Jodid einen merklichen Einfluß auf den thermischen Effekt ausüben könnte.

Es war zu vermuten, daß das tertiäre Amin bei den Versuchen merklich auf den Überschuß von Jodid einwirken werde, doch eine unmittelbare Untersuchung dieser Frage bewies das Gegenteil: in die calorimetrische Kammer wurde Propyljodid in Benzollösung eingeführt, und dann wurden die zu den Versuchen verwendeten Amine hinzugegossen, wobei nur eine nichtssagende Verlangsamung im Temperaturabfall während 1-2 Minuten bemerkt wurde, nach welcher die Temperatur fortfuhr, regelmäßig in vorigen Tempo zu sinken, was 15 Minuten und länger dauerte. Als ich mich davon überzeugt hatte, vermutete ich, daß sich der Vorgang in Anwesenheit ron $\mathrm{C}_{3} \mathrm{H}_{7} \mathrm{Mg} \mathrm{J}$ ăndern könnte; jedoch überzeugten mich abermals unmittelbare Versuche unter Einführung geringer Mengen von $\mathrm{C}_{3} \mathrm{H}_{7} \mathrm{MgJ}$ in die Kammer, $\mathrm{da} ß$ auch die magnesiumorganische Verbindung keine merkliche gegenseitige Einwirkung von Jodid und Amin hervorruft. Dadurch wurde auch die gefaßte Vermutung beseitigt. Also, obgleich tertiäre Amine zweifellos auf Jodide reagieren, so ist doch dieGeschwindigkeit dieser Reaktion unter den Versuchsbedingungen eine so geringe, daß man sie praktisch mit vollem Reeht vollkommen ignorieren konnte.

Die zu den Versuchen verwendeten tertiären Amine wurden vor den Versuchen sorgfältig über metallischem Natrium destilliert, wobei Fraktioneu von dem jeweiligen Amin streng entsprechender Temperatur gesammelt wurden.

1) Diese Berichte 38, 3666 [1905]; Journ. d. Russ. Phys.-chem. Ges. 37, 1104. 
Bei Einwirkung auf eine magnesiumorganische Verbindung schieden sie gar keine Gasbläschen aus, was überhaupt als guter Beweis ihrer Reinheit dienen kann.

Was endlich das Benzol anbetrifft, so ist es vor den Versuchen ebenfalls aber metallischęm Natrium destilliert worden.

In denjenigen Fällen, wo tertiäre Amine, die ihrer Konstitution nach ausschließlich die einfachsten Radikale enthielten, zu den Versuchen mit Benzol dienten, erhielt man nach Beendigung der Operation eine dickflüssige Lösung, da die sich bildenden Aminkomplexe in Benzol leicht löslich sind; im Gegensatz hierzu bildeten sich bei höheren fetten (mit dem Radikal $\mathrm{C}_{5} \mathrm{H}_{11}$ ), besonders aber bei aromatischen Aminen - genauer bei fettaromatischen, denn ich nahm Amine, in denen nur das eine von drei Radikalen aromatisch war - Körper, die entweder unlöslich in Benzol oder nur wenig darin löslich waren.

Die Versuche selbst wurden folgendermaßen angestellt: Nach der Zubereitung der individuellen magnesiumorganischen Verbindung, unmittelbar in derselben Kammer, worin der Versuch angestellt werden sollte, worde tertiäres Amin direkt in dem Tropftrichter abgewogen, aus welchem es während des Versuches herausfloB. Die Amine nahm ich immer in fast genau theoretischer Menge, übrigens ändert ein Überschuß davon nichts wesentliches am Vorgange, denn es tritt immer nur ein Molekül Amin in Reaktion. Nach der Aufstellung der Kammer im Calorimeter und zehn Zwischenpausen (5 Min.) der Anfangsperiode, wurde die ganze Menge tertiäres Amin in Benzollösung aut ein Mal in die Kammer eingeführt und die Reaktionsmischung mit dem Platinrührer sorgfältig durchgemengt. Der Tropftrichter wurde danach mit einer abgewogenen Quantität absoluten Benzols ausgespült, wodurch die letzten Mengen Amin in die Kammer gebracht wurden. Bei diesen Bedingungen währte die Hauptperiode 4-6 Minuten; nachdem dann noch 10 Ablesungen vorgenommen worden waren, welche die Schlußperiode bildeten, galten die Tersuche als beendet.

Die Resultate dieser Versuche sind in folgenden sechs Tabellen dargestellt, worin angezeigt sind: 1. die Mengen von Magnesium, Jodid und Benzol, die zur Zubereitung der individuellen magnesiumorganischen Verbindungen dienten; 2. die Menge Amin, die zum Versuch genommen wurde, wobei in den ersten drei Tabellen die Versuche mit Aminen der Fettreihe dargestellt sind: Triäthylamin, Tripropylamin und Tri-i-anylamin, und in den folgenden drei die Versuche mit Aminen der aromatischen Reihe: Dimethylanilin, Dimethyl-o-toluidin und Diäthylanilin; 3. der Wasserwert des calorimetrischen Systems $=\mathrm{W} ; 4$. die Temperatursteigerung mit Korrektur auf Wärmestrahlung $t_{n}-t_{0}\left(+t_{r}\right) ; 5$. der Wärmeüberschuß, der mit dem tertiären Amin und mit dem zı seiner Auflösung benutzten Benzol eingeführt worden ist $=\tau$; endlich 6 . der thermische Effekt $Q$, der unmittelbar für die gegebene Menge individueller magnesiumorganischer Verbindungen in 
kleinen Cal. aufgefunden, so auch für ein Grammmol. in großen Cal. und in Kilojoulen ausgerechnet worden ist.

Tabelle 1.

$\mathrm{li} \mathrm{MgJ}+\left(\mathrm{C}_{2} \mathrm{H}_{5}\right)_{3} \mathrm{~N}$.

\begin{tabular}{|c|c|c|c|c|c|c|c|c|c|c|}
\hline \multirow{3}{*}{ 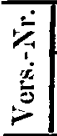 } & \multicolumn{3}{|c|}{$\mathrm{RMg} J$} & \multirow{3}{*}{ 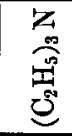 } & \multirow{3}{*}{ W } & \multirow{3}{*}{5} & \multirow{3}{*}{$\tau$} & \multicolumn{3}{|c|}{$\mathrm{Q}$} \\
\hline & \multirow{2}{*}{$\mathrm{Mg}$} & \multirow{2}{*}{$\mathrm{C}_{3} \mathrm{H}_{\mathrm{i}} \mathrm{J}$} & \multirow{2}{*}{$\mathrm{C}_{6} \mathrm{H}_{6}$} & & & & & \multirow{2}{*}{$\begin{array}{c}\text { Vers.- } \\
\text { Cal. }\end{array}$} & \multicolumn{2}{|c|}{ Gr.-Mrol. } \\
\hline & & & & & & & & & Cal. & $\bar{J}$ \\
\hline $\begin{array}{l}1 \\
2\end{array}$ & $\begin{array}{l}0.6334 \\
0.5747\end{array}$ & $\begin{array}{l}7.8 \\
7.0\end{array}$ & $\begin{array}{l}13.1 \\
12.3\end{array}$ & $\begin{array}{l}2.72 \\
2.47\end{array}$ & $\begin{array}{l}743.3 \\
741.1\end{array}$ & $\begin{array}{l}0.4146 \\
0.3780\end{array}$ & $\begin{array}{c}0 \\
-0.4\end{array}$ & $\begin{array}{l}308.2 \\
280.5\end{array}$ & $\begin{array}{l}11.68 \\
11.71\end{array}$ & $\begin{array}{l}48.8 \\
490\end{array}$ \\
\hline & & & & & & & & & 11.7 & 48.94 \\
\hline
\end{tabular}

Tabelle II.

$\mathrm{RMgJ}+\left(\mathrm{C}_{3} \mathrm{H}_{7}\right)_{3} \mathrm{~N}$.

\begin{tabular}{|c|c|c|c|c|c|c|c|c|c|c|}
\hline \multirow{3}{*}{$\begin{array}{l}\dot{\bar{*}} \\
1 \\
\dot{p} \\
2 \\
2\end{array}$} & \multicolumn{3}{|c|}{$\mathrm{RMg} \mathrm{J}$} & \multirow{3}{*}{ 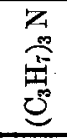 } & \multirow{3}{*}{ W } & \multirow{3}{*}{$\stackrel{5}{1+5}$} & \multirow{3}{*}{$\tau$} & \multicolumn{3}{|c|}{$\bar{Q}$} \\
\hline & \multirow{2}{*}{$\mathbf{M g}$} & \multirow{2}{*}{$\mathrm{C}_{3} \mathrm{H}_{7} \mathrm{~J}$} & \multirow{2}{*}{$\mathrm{C}_{6} \mathrm{H}_{6}$} & & & & & \multirow{2}{*}{$\begin{array}{c}\text { Vers.- } \\
\text { Cal. }\end{array}$} & \multicolumn{2}{|c|}{ Gr.-Mol. } \\
\hline & & & & & & & & & Cal. & $\mathrm{J}$ \\
\hline 3 & $\begin{array}{l}0.6198 \\
0.6158\end{array}$ & $\begin{array}{l}7.3 \\
7.1\end{array}$ & $\begin{array}{l}14.9 \\
15.6\end{array}$ & $\begin{array}{l}3.73 \\
3.70\end{array}$ & $\begin{array}{l}742.5 \\
743.2\end{array}$ & $\begin{array}{l}0.3636 \\
0.3518\end{array}$ & $\begin{array}{c}0 \\
-0.1\end{array}$ & $\begin{array}{l}269.9 \\
261.5\end{array}$ & $\begin{array}{l}10.45 \\
10.19 \\
\end{array}$ & $\begin{array}{l}43.71 \\
42.63 \\
\end{array}$ \\
\hline
\end{tabular}

Tabelle IlI.

$\mathrm{R} \mathrm{NgJ}+\left(\mathrm{C}_{5} \mathrm{H}_{11}\right)_{3} \mathrm{~N}$.

\begin{tabular}{|c|c|c|c|c|c|c|c|c|c|c|}
\hline \multirow{3}{*}{ 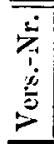 } & \multicolumn{3}{|c|}{$\mathrm{RMg} \mathrm{J}$} & \multirow{3}{*}{ 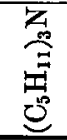 } & \multirow{3}{*}{ w } & \multirow{3}{*}{$\left.\right|_{5} ^{+\Xi}$} & \multirow{3}{*}{$\boldsymbol{\tau}$} & \multicolumn{3}{|c|}{$\mathbf{Q}$} \\
\hline & \multirow{2}{*}{$\mathrm{MIg}$} & \multirow{2}{*}{$\mathrm{C}_{3} \mathrm{H}_{7} \mathrm{~J}$} & \multirow{2}{*}{$\mathrm{C}_{6} \mathrm{H}_{6}$} & & & & & \multirow{2}{*}{$\begin{array}{l}\text { Vers.- } \\
\text { Cal. }\end{array}$} & \multicolumn{2}{|c|}{ Gr.-Mol. } \\
\hline & & & & & & & & & Cal. & $\overline{\mathrm{J}}$ \\
\hline 5. & 0.6255 & 8.3 & 11.9 & 6.00 & 746.5 & 0.3083 & -1.8 & 231.9 & 8.90 & 37.23 \\
\hline & 6344 & 7.4 & 13.4 & 6.21 & 742.3 & 10 & $1+0.9$ & 240.4 & 9.09 & 38.02 \\
\hline
\end{tabular}

Tabelle IV.

$\mathrm{RMgJ}+\mathrm{C}_{6} \mathrm{H}_{5} \cdot \mathrm{N}\left(\mathrm{CH}_{3}\right)_{2}$.

\begin{tabular}{|c|c|c|c|c|c|c|c|c|c|c|}
\hline \multirow{3}{*}{ 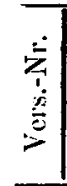 } & \multicolumn{3}{|c|}{ RMg J } & \multirow{3}{*}{ 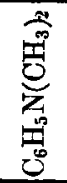 } & \multirow{3}{*}{ W } & \multirow{3}{*}{$\stackrel{=}{ \pm}$} & \multirow{3}{*}{$\boldsymbol{\tau}$} & \multicolumn{3}{|c|}{ Q } \\
\hline & \multirow{2}{*}{$\mathrm{Mg}$} & \multirow{2}{*}{$\mathrm{C}_{3} \mathrm{H}_{7} \mathrm{~J}$} & \multirow{2}{*}{$\mathrm{C}_{6} \mathrm{H}_{6}$} & & & & & \multirow{2}{*}{$\begin{array}{c}\text { Vers.- } \\
\text { Cal. }\end{array}$} & \multicolumn{2}{|c|}{ Gr.-Mol. } \\
\hline & & & & & & & & & Cal. & $\mathrm{J}$ \\
\hline $\begin{array}{l}7 \\
8\end{array}$ & $\begin{array}{l}0.6167 \\
0.6106\end{array}$ & $\begin{array}{l}7.2 \\
7.6\end{array}$ & $\begin{array}{l}13.7 \\
12.9\end{array}$ & $\begin{array}{l}3.17 \\
3.10\end{array}$ & $\begin{array}{r}742.2 \\
7+3.1\end{array}$ & $\begin{array}{l}0.1308 \\
0.1313\end{array}$ & $\begin{array}{l}0 \\
0\end{array}$ & $\begin{array}{l}97.1 \\
976\end{array}$ & $\begin{array}{l}3.78 \\
3.83\end{array}$ & 15.81 \\
\hline & & & & & & & & & 3.81 & 15. \\
\hline
\end{tabular}


Tabelle V.

$\mathrm{RMg} \mathrm{J}+\mathrm{CH}_{3}^{-} \cdot \mathrm{C}_{6} \mathrm{H}_{4} \cdot \mathrm{N}\left(\mathrm{CH}_{3}\right)_{2}$.

\begin{tabular}{|c|c|c|c|c|c|c|c|c|c|c|}
\hline \multirow{3}{*}{ 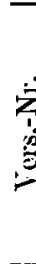 } & \multicolumn{3}{|c|}{$\operatorname{lMg} \mathrm{T}$} & \multirow{3}{*}{ 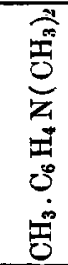 } & \multirow{3}{*}{$\mathbf{W}$} & \multirow{3}{*}{ 吾 } & \multirow{3}{*}{$\boldsymbol{\tau}$} & \multicolumn{3}{|c|}{$Q$} \\
\hline & \multirow{2}{*}{$\mathrm{Mg}$} & \multirow{2}{*}{$\mathrm{C}_{3} \mathrm{H}_{7} \mathrm{~J}$} & \multirow{2}{*}{$\mathrm{C}_{6} \mathrm{H}_{6}$} & & & & & Vers.- & \multicolumn{2}{|c|}{ Gr.-Mol. } \\
\hline & & & & & & & & & Cal. & $\mathrm{J}$ \\
\hline $\begin{array}{r}9 \\
10\end{array}$ & $\begin{array}{l}0.6100 \\
0.6255\end{array}$ & $\begin{array}{l}7.2 \\
7.9\end{array}$ & $\begin{array}{l}13.3 \\
12.7\end{array}$ & $\begin{array}{l}3.48 \\
3.58\end{array}$ & $\begin{array}{l}742.6 \\
744.8\end{array}$ & $\begin{array}{l}0.0340 \\
0.0351\end{array}$ & $\mid \begin{array}{c}0.8 \\
0\end{array}$ & $\begin{array}{l}24.5 \\
26.1\end{array}$ & $\begin{array}{l}0.96 \\
1.00 \\
\end{array}$ & $\begin{array}{l}4.02 \\
4.18 \\
\end{array}$ \\
\hline
\end{tabular}

Tabelle VI.

$\mathrm{RMgJ}+\mathrm{C}_{6} \mathrm{H}_{5} \cdot \mathrm{N}\left(\mathrm{C}_{2} \mathrm{H}_{5}\right)_{2}$.

\begin{tabular}{|c|c|c|c|c|c|c|c|c|c|c|}
\hline \multirow{3}{*}{$\begin{array}{l}\dot{3} \\
\dot{8} \\
\frac{1}{3}\end{array}$} & \multicolumn{3}{|c|}{ RMg J } & \multirow{3}{*}{ 苞 } & \multirow{3}{*}{ W } & \multirow{3}{*}{$\begin{array}{l}= \pm \\
1 \pm t\end{array}$} & \multirow{3}{*}{$\tau$} & \multicolumn{3}{|c|}{$\mathbf{Q}$} \\
\hline & \multirow{2}{*}{$\mathrm{Mg}$} & \multirow{2}{*}{$\mathrm{C}_{3} \mathrm{H}_{7} \mathrm{~J}$} & \multirow{2}{*}{$\mathrm{C}_{6} \mathrm{H}_{6}$} & & & & & Vers.- & \multicolumn{2}{|c|}{ Gr.-Mol. } \\
\hline & & & & & & & & & Cal. & J \\
\hline $\begin{array}{l}11 \\
12\end{array}$ & $\begin{array}{l}0.6300 \\
0.6254\end{array}$ & $\begin{array}{l}6.9 \\
7.4\end{array}$ & $\begin{array}{l}13.6 \\
13.2\end{array}$ & $\begin{array}{l}3.98 \\
3.95\end{array}$ & $\begin{array}{l}744.0 \\
743.0\end{array}$ & $\begin{array}{l}0.0281 \\
0.0274\end{array}$ & $\begin{array}{l}0 \\
0\end{array}$ & $\begin{array}{l}20.9 \\
20.4\end{array}$ & $\begin{array}{l}0.79 \\
0.78\end{array}$ & $\begin{array}{l}3.30 \\
3.26\end{array}$ \\
\hline & & & & & & & & & 0.78 & 3.28 \\
\hline
\end{tabular}

SchluB.

Die angefïhrten Versuche beweisen, daß die individuellen magnesiumorganischen Verbindungen fähig sind, mit tertiären Aminen Komplexe zu bilden, die den Monoätheraten vollständig analog sind, und dabei mebr oder weniger bedeutende Wärmemengen ausscheiden, jedoch, unter gewöhnlichen Bedingungen wenigstens, keine den Diätheraten analogen Komplexe geben.

Angesichts des Umstandes, daß Komplexverbindungen mit tertiäreu Aminen verhältnismäßig sehr selten sind ${ }^{1}$ ), gewinnen die untersuchten Komplexe ein besonderes Interesse, indem sie noch außerdem auf eine erhöhte Fähigkeit der individuellen magnesiumorganischen Yerbinclungen zur Bildung von Komplexverbindungen ïberhaupt hinweisen.

Was ferner den Einfluß der Radikale auf den thermischen Effekt der Anlagerung der tertiären Amine und folglich auch im allgemeinen auf die Natur des Stickstoffatoms anbetrifft, so läßt sich aus den er-

1) Vergl. »Untersuchungen in dem Geljiete der Komplexverbindungen« von I. 'Tschugaeff, S. 140 . 
haltenen Zahlen ersehen, daß mit der Vergrößerung der Masse der Radikale in der Fettreihe die Natur des Stickstoffatoms sich in dem Sinne verändert, daß der thermische Effekt der Anlagerung der Amine langsam fällt; bei dem Übergang von fetten zu aromatischen Aminen läßt sich sogleich der starke negative Einfluß der aromatischen Radikale beobachten, der in einem starken Fallen des thermischen Effekts zum Ausdruck kommt und fast bis auf Null sinkt. Wenn man den Einfluß der Radikale auf das Stickstoffatom mit dem Einfluß derselben auf das Sauerstoffatom ${ }^{1}$ ) vergleicht, soweit sich darauf aus dem thermischen Effekt der Anlagerung der Äther und tertiären Amine an eine und dieselbe magnesiumorganische Verbindung $\left(\mathrm{C}_{3} \mathrm{H}_{7} \mathrm{MgJ}\right)$ schließen läßt, so kann man darin einen beachtenswerten Parallelismus bemerken, was aus folgender Zusammenstellung $\mathrm{zu}$ ersehen ist:

\begin{tabular}{|c|c|c|c|c|c|c|c|}
\hline Oxonit & a-Komple & & & Ammoni & $\mathrm{m}-\mathrm{Ko}$ & mplexe & \\
\hline${ }_{2} \mathrm{H}_{\tilde{3}} . \mathrm{O} . \mathrm{C}_{2} \mathrm{H}_{\tilde{\mathbf{s}}}$ & & 6.63 & & $\left(\mathrm{C}_{2} \mathrm{H}_{5}\right)_{3} \mathrm{~N}$ & & 11.70 & \\
\hline $\mathrm{C}_{2} \mathrm{H}_{5}$ & . & 6.15 & " & $\left(\mathrm{C}_{3} \mathrm{H}_{7}\right)_{3} \mathrm{~N}$ & & 10.32 & \\
\hline 0. $\mathrm{C}_{2} \mathrm{H}_{5}$ & & 5.91 & » & $\left(\mathrm{C}_{5} \mathrm{H}_{11}\right)_{3} \mathrm{~N}$ & & 9.00 & \\
\hline $\mathrm{C}_{6} \mathrm{H}_{\mathrm{j}}, 0 . \mathrm{CH}_{3}$ & nahezu & 0 & $»$ & $\mathrm{C}_{6} \mathrm{H}_{5} \cdot \mathrm{N}\left(\mathrm{CH}_{3}\right)_{2}$ & & 3.81 & \\
\hline $\mathrm{C}_{6} \mathrm{H}_{5} .0 . \mathrm{C}_{2} \mathrm{H}_{\overline{5}}$ & nahezu & 0 & $»$ & $\mathrm{C}_{6} \mathrm{H}_{5} \cdot \mathrm{N}\left(\mathrm{C}_{2} \mathrm{H}_{5}\right)_{2}$ & & 0.78 & \\
\hline
\end{tabular}

Aus einer Vergleichung der Zahlen der wagerechten Reihen läßt sich auch ersehen, da $\beta$ bei der Bildung der Ammoniumverbindungen mehr Wärme ausgeschieden wird, als bei der Bildung entsprechender Oxoniumverbindungen. Diese Folgerung konnte in qualitativer Hinsicht auch vorausgesehen werdep auf Grund alles dessen, was uns über Ammonium- und Oxoniumverbindungen bekannt ist; aber die angeführte Tabelle gibt außerdem quantitative Verhältnisse an, und zwar in Anwendung auf - in chemischem Sinne - so leichtbewegliche Verbindungen, wie sie in den magnesiumorganischen vorliegen, und in einem Medium, worin die Ionisierung ausgeschlossen ist.

Zum Schluß muß bemerkt werden, $\mathrm{da} \beta$ die angeführten Untersuchungen erst möglich wurden, nachdem die individuellen magnesiumorganischen Verbindungen erhalten worden sind, welch letztere infolge ihrer originellen Stellung in der Reihe der Salze, wie ich hoffe, die Möglichkeit geben werden, auch noch einige andere Grundfragen aus dem Gebiet der Komplexverbindungen zu beleuchten.

Es sei mir gestattet, an dieser Stelle Frl. C. J. Hesse für ihre Teilnahme an der Zusammenstellung dieser Abhandlung meinen besten Dank auszusprechen.

Moskau, Chem. Universitätslaboratorium. 26. Dezember 1906.

y) Compt. rend. 143, 1237 [1906]. 